more attractive or by making the competition less attractive (Buss \& Dedden 1990; Schmitt \& Buss 1996). A common method of making someone appear less attractive is to derogate, slight, and insult them. Buss and Dedden (1990) found that men and women slighted and insulted exactly those qualities that would have been critical to survival and reproductive success in evolutionary history. Men derogated other men's manhood, ambition, achievements, and strength. Women criticized other women's physical appearance, and implied either that they were promiscuous or that they were sexual teases. The authors also found that women were more likely than men to insult competitors' intelligence (although they did not predict this finding). A study of sexual competition among female medical students suggests that this practice may result from two causes (Townsend 1998). First, it makes tactical sense to criticize competitors on traits in which the competitors appear weak, for example, an intellectual but plain woman whose ex-boyfriend is going out with a fashion model would be foolish to put down her competitor's looks. This would only draw attention to her competitor's superiority and her own deficiencies in that area. Instead, she would probably slight her rival's intelligence perhaps also using her opponent's youth and physical superiority as proof of her intellectual inferiority. A second reason professional women might derogate a competitor's intelligence is that their own criteria for attractiveness - which are largely socioeconomic and include intelligence (Kenrick et al. 1990) - seem natural and right (Townsend 1998). Consequently, criteria that men favor such as youth and beauty often seem foreign, incomprehensible, and degraded to women. Numerous women in the study deprecated female competitors with lower SES by calling them bimbos, airheads, and dingalings (Townsend 1998). These women also derogated male peers who dated women with lower SES particularly if those women were younger. Similarly, organizational limitations on dating between men and their status inferiors may serve the ostensible function of protecting against sexual harassment, but they also tend to limit the ability of lower-status women (who are often younger and therefore more sexually attractive to men) to compete with higher-status women for higherstatus men.

In her conclusion, Campbell writes that patriarchal control has caused female aggression to be viewed as unnatural, and this taboo causes women to excuse rather than justify their aggression. She argues cogently that in ancestral populations direct aggression did not produce the reproductive payoffs that it did for men; consequently, compared to male aggression, female aggression is more likely to be low-level and indirect. If this is true, direct aggression in females would presumably fit better with expressive theories than instrumental theories, and expressive and instrumental theories correspond respectively to excuses and justifications (sect. 2.2). If the sex difference in the use of excuses and justifications remains, even when indirect forms of aggression are considered (Archer \& Parker 1994), then women's greater tendency to use excuses is perhaps an example of cultural enhancement of an evolutionarily based sex difference, but it is not solely a cultural construction imposed by patriarchy. Similarly, phrases like "the myth of the coy female" and "the myth of the nonaggressive women" can be politically useful (sect. 3, Conclusions), but they can also be misleading. It is important to deconstruct the myths of the coy and nonaggressive woman, but it is equally important to deconstruct the myth of the absence of evolutionarily based sex differences in behavior. Campbell's target article does an admirable job of this.

\section{How is maternal survival related to reproductive success?}

\section{T. Wang and Ralph Hertwig \\ Center for Adaptive Behavior and Cognition, Max Planck Institute for Human Development, 14195 Berlin, Germany. xtwang@usd.edu hertwig@mpib-berlin.mpg.de}

Abstract: Campbell's target article is a stimulating attempt to extend our understanding of sex differences in risk-taking behaviors. However, Campbell does not succeed in demonstrating that her account adds explanatory power to those (e.g., Daly \& Wilson 1994) previously proposed. In particular, little effort was made to explore the causal links between survival (staying alive) and reproduction.

As Darwin $(1859$; 1871) noted, evolution is a matter of differential reproduction rather than differential survival. For human males, reproductive success and personal survival is often a tradeoff. The greater variance in reproductive success among males selects for greater acceptance of risk in male-male competition; according to Daly and Wilson (1994; Wilson \& Daly 1985), this is why males are more risk seeking than females. In contrast, for human females, reproductive success and personal survival tend to be interdependent goals. Thus, according to Campbell's "staying alive" account, natural selection would favor risk avoidance in females because infant survival depends more on maternal than on paternal care and defense.

Both accounts mentioned attempts to explain sex differences in the acceptance of risk, in particular with regard to aggression. Daly and Wilson attribute these differences to male adaptation to the greater variance in reproduction, whereas Campbell suggests two distinct mental adaptations, one for men and one for women. Is the additional female adaptation necessary? In our view, Campbell fails to convince the reader that it is.

As Campbell points out, there are four important empirical facts that any adequate theory of male and female patterns of aggression have to explain. The first two facts - that human males engage in aggression more frequently than females, and that this sex difference increases as a function of increasing seriousness of the aggressive behavior - are well captured by Daly and Wilson's account. The third fact is a high correlation between rates of male and female aggression across geographical areas. Campbell explains it in terms of a mutual response to the same ecological conditions (e.g., resource shortage) - an argument which seems to be extraneous to both accounts. Finally, the fourth fact - a high correlation between rates of male and female aggression over the life span - appears to be inconsistent with Campbell's account.

What is behind the age-related patterns of criminal violence? In both sexes, criminal violence is most likely to occur between the ages of 14 to 24 . One explanation of this high correlation is that the intrasex competition for mates becomes most intensified after puberty for both males and females. However, according to the "staying alive" hypothesis, we could expect that females, especially in hunter and gatherer societies, would be most risk-avoiding at this life stage, when they are most fecund and most likely to be involved in maternal care and defense. Given Campbell's claim that this female adaptation occurs because the mother's survival is critical for her own reproductive success, the finding that criminal violence by females peaks at this life stage is unexpected. In order to explain this phenomenon, Campbell simply switches to a mating resource competition account: "The rise in female aggression during adolescence, like that of males, is associated with mate selection."

Future research on whether there is a significant age effect upon risk acceptance and criminal violence may provide the data with which to judge whether or not a female risk-avoidance adaptation is required. For instance, a testable prediction from Campbell's "staying alive" hypothesis might be that women near or after menopause become more risk seeking because both their own reproduction and offspring's survival are less dependent upon their personal survival. 
What links maternal survival to reproductive success? The pivotal point of the "staying alive" hypothesis is that maternal care is more important for offspring survival than paternal care. Why is this the case? What are the causal links between maternal survival and reproductive success? Had Campbell tried to search for possible causal links, the "staying alive" hypothesis could have been tested more rigorously. We can think of at least three possible causal links: (1) The maternal proportion of parental investment is greater than the paternal proportion. (2) Given the close physical proximity between a mother and her infant, risk avoidance by the mother will enhance the survival chances of her offspring, thereby enhancing her own reproductive success. (3) The kinds of parental care that mothers give (e.g., breast feeding) are more crucial for infant survival than the kinds of care given by fathers (e.g., protecting both mother and offspring from the dangers imposed by predators). However, the third postulate is open to the argument that paternal protection is more crucial than maternal feeding for infant survival.

The causal links identified here, while tentative, can be systematically tested. The sex differences in aggression, for instance, can be examined under two comparable conditions where a proposed causal relation is reversed or differs across conditions. For example, considering the relative amount of parental investment, one way to conduct such a test would be to compare female risk taking or aggression patterns under both high and low paternal investment conditions. If the relative amount is the crucial causal factor, then female risk taking should be lower in low paternal investment conditions where maternal care and defense are more important for offspring survival. This logic of hypothesis testing with a reversed causal structure has been successfully applied by George Williams (1966) and Robert Trivers (1972) in testing their evolutionary hypotheses. Similarly, if physical proximity is the crucial causal factor, one could examine whether female risk taking changes with varying degrees of physical proximity between mother and infant. Such analyses would make Campbell's theoretical claims empirically testable.

As we suggest, there are several possible ways to examine Campbell's theoretical claims. At this point, we are left with a stimulating hypothesis which attempts to incorporate the existing literature in the area of sex differences in risk-taking behaviors; however, without demonstrating the causal links between maternal survival and reproductive success, we can say little about the theoretical and empirical validity of this hypothesis.

\section{Author’s Response}

\section{The last days of discord? Evolution and culture as accounts of female-female aggression}

\section{Anne Campbell \\ Psychology Department, Durham University, Durham DH1 3LE, England. a.c.campbell@durham.ac.uk}

\begin{abstract}
When aggression is conceptualised in terms of a costbenefit ratio, sex differences are best understood by a consideration of female costs as well as male benefits. Benefits must be extremely high to outweigh the greater costs borne by females, and circumstances where this occurs are discussed. Achievement of dominance is not such a circumstance and evidence bearing upon women's egalitarian relationships is reviewed. Attempts to explain sex differences in terms of sexual dimorphism, sex-of-target effects, social control, and socialisation are found to be inadequate. The suggestion that the stigmatisation of female aggreession arises
\end{abstract}

not from patriarchal imposition but from statistical rarity (resulting from evolutionary pressures) is given serious consideration. Two hypotheses ("internal read-out" versus social/epidemiological representations) are described to explain the relationship between sex differences in behaviour and corresponding lay explanations.

It is a rare treat to have a series of world renowned researchers climb into the academic boxing ring for 27 rounds with my proposals. It is especially gratifying because debating bouts on female aggression are usually relegated to the equivalent of the local youth club rather than an international venue. Because most commentators were kind enough to keep their gloves on, I am unbloodied, if slightly bowed, as I take this opportunity to respond. My thanks to all the commentators for the time and thought that they devoted to evaluating and refining the ideas expressed in my target article. Though unable to address every point raised, I have grouped the main issues into nine areas of controversy.

\section{R1. Do we need a second mechanism for sex differences in aggression?}

The impact of Daly and Wilson's (1988a) seminal analysis of male violence has been immense - and rightly so. Although some commentators take issue with their analysis (Beckerman queries the relationship between male dominance and reproductive success, Cashdan queries the domain-generality and sex-specificity of competitiveness), Mealey seems to believe that I am merely rehearsing their position, whereas others, Simpson, Brain, and Archer, question whether my additions to their model are really necessary.

Following Alexander's (1979, p. 241) notion that "the entire life history strategy of males is a higher-risk, higherstakes adventure than that of females," Daly and Wilson (1990) pursue these twin concepts by treating risk as variance in the magnitude of the payoff for a given course of action and stakes as the possible gains. Hence their analysis focuses heavily, though not exclusively, upon the positive incentives for aggression by males ("males typically compete for bigger prizes than do females. Bigger prizes warrant bigger gambles," Daly \& Wilson 1988a, p. 163). Stakes, however, refer more properly to the magnitude of the bet that is made (and hence to how much can be lost) and risk usually refers to the likelihood of losing (we do not normally speak of the risk of winning the lottery but may refer to the risk of losing our stake). It was this observation that prompted me to think that women had more to lose than men in terms of reproductive success and to wonder whether women might therefore avoid risky situations. The burden of my argument is that women actively seek to avoid direct combat and, where this is impossible, to minimise its lethality or to use indirect means of competition. Cashdan argues that a focus on male payoffs predicts greater male competitiveness in general, while an emphasis upon female costs predicts only that female competition will be less intense than that of males. She believes, and I concur, that the latter prediction is better borne out by the data.

So what began as semantic hair-splitting turned into a new way of looking at sex differences in aggression. But do we need it? While Daly and Wilson emphasise the benefits of aggression for males, I emphasise the costs for females. 